\title{
A Meshless Method with Radial Basis Function for Solving Unsaturated Flow in Heterogeneous Porous Media
}

\author{
Cheng-Yu Ku \\ Department of Harbor and River Engineering, National Taiwan Ocean University, Keelung, Taiwan \\ Chih-Yu Liu \\ Department of Harbor and River Engineering, National Taiwan Ocean University, Keelung, Taiwan, \\ 20452003@email.ntou.edu.tw \\ Wei-Po Huang \\ Department of Harbor and River Engineering, National Taiwan Ocean University, Keelung, Taiwan \\ Jing-En Xiao \\ Department of Harbor and River Engineering, National Taiwan Ocean University, Keelung, Taiwan
}

Follow this and additional works at: https://jmstt.ntou.edu.tw/journal

Part of the Fresh Water Studies Commons, Marine Biology Commons, Ocean Engineering Commons, Oceanography Commons, and the Other Oceanography and Atmospheric Sciences and Meteorology Commons

\section{Recommended Citation}

Ku, Cheng-Yu; Liu, Chih-Yu; Huang, Wei-Po; and Xiao, Jing-En (2021) "A Meshless Method with Radial Basis Function for Solving Unsaturated Flow in Heterogeneous Porous Media," Journal of Marine Science and Technology. Vol. 29: Iss. 5, Article 10.

DOI: $10.51400 / 2709-6998.2470$

Available at: https://jmstt.ntou.edu.tw/journal/vol29/iss5/10

This Research Article is brought to you for free and open access by Journal of Marine Science and Technology. It has been accepted for inclusion in Journal of Marine Science and Technology by an authorized editor of Journal of Marine Science and Technology. 


\title{
A Meshless Method with Radial Basis Function for Solving Unsaturated Flow in Heterogeneous Porous Media
}

\author{
Cheng-Yu Ku ${ }^{\text {a,b }}$, Chih-Yu Liu ${ }^{\text {a,*, }}$, Wei-Po Huang ${ }^{\text {a,b }}$, Jing-En Xiao ${ }^{a}$ \\ a Department of Harbor and River Engineering, National Taiwan Ocean University, Keelung, 20224, Taiwan \\ ${ }^{\mathrm{b}}$ Center of Excellence for Ocean Engineering, National Taiwan Ocean University, Keelung, 20224, Taiwan
}

\begin{abstract}
This paper presents a study for solving unsaturated flow in heterogeneous porous media using the meshless method with the radial basis function (RBF). For modeling the nonlinear hydrological process in unsaturated zone, an exponential model is introduced in the Richards equation such that we may obtain the linearized Richards equation. We adopt the multiquadric function as the RBF in the meshless method for solving the linearized Richards equation. For simulating the unsaturated flow problems in layered heterogeneous soils, the flux and the head must satisfy the continuity condition at the interface. Several examples are carried out for modeling the hydrological process in multi-layered unsaturated soils. The results demonstrate that we only discretize by a set of points without tedious mesh generation and significantly enhance the applicability for solving unsaturated flow problems, especially in heterogeneous multi-layered soils with extreme physical property contrasts.
\end{abstract}

Keywords: Unsaturated flow, Richards equation, Radial basis function, Meshless method, Heterogeneous porous media

\section{Introduction}

$\mathrm{T}$ he modeling of flow in vadose zone is often encountered in a variety of groundwater flow, hydrogeology, and geotechnical engineering applications [8,17]. Due to the characteristic of soil water interaction as well as unsaturated soil permeability, flow movement in unsaturated porous media is a highly nonlinear hydrological process [6]. Based on the continuous equation and Darcy-Buckingham law, the nonlinear hydrological process in unsaturated porous media may refer to the Richards equation [35]. Since it is one of the most complicated hydrological processes in nature, analytical solutions of Richards equation only exist for simplified examples [3,34]. Thus, practical engineering problems and applications require numerical solutions in one, two or three dimensions $[4,9,30]$.
Several numerical approaches based on the mesh-based methods to the modeling of flow movement in unsaturated porous media have been proposed, such as the boundary element method [26], the finite difference method (FDM) [20,21], and the finite element method [31,33]. Even though the success of mesh-based methods is effective and easily implemented for dealing with unsaturated flow problems, limitations still remain while utilizing the mesh-based methods including mesh-generation for complex geometries, or short time interval for the numerical convergence. As a result, several computational approaches based on the meshless scheme have drawn considerable attention and been proposed, such as the Trefftz method [15], the method of fundamental solutions [13], the spacetime collocation meshless method [22], the local radial basis function (RBF) based 
differential quadrature method [1,25], and the RBF collocation method $[11,16,19,23]$. The meshless method using RBF is probably one of the common used methods for dealing with boundary or initial value problems where the variables could be expressed by using the function approximation $[12,18,29,37]$. The RBF collocation method was firstly proposed by Kansa for solving different kinds of partial differential equation including elliptic, parabolic, and hyperbolic partial differential equations [14]. Recently published works show that the RBF collocation method is an efficient numerical method to deal with the heat equation, wave equation, saturated and unsaturated flow problems. For instance, Dehghan and Shokri have developed the RBF collocation method for solving the nonlinear Klein-Gordon equation [5]. The local RBF collocation method for linear thermoelasticity in two dimensions has also been proposed and applied for dealing with compressible Navier-Stokes equations as well as advection-diffusion problems [23,25]. Moreover, the RBF collocation method for simulating the unsaturated seepage problems have been developed [35]. Generally, the hydrological process in layered porous media is much more common than homogeneous porous media [2,24]. Although considerable research has been devoted to the development of the RBF collocation method, rather less attention has been paid to the modeling of two-dimensional hydrological process in unsaturated heterogeneous multi-layered porous media.

In this article, we propose a study for solving unsaturated flow in heterogeneous porous media using the novel meshless method with the RBF. For modeling the nonlinear hydrological process in unsaturated zone, an exponential model is introduced in the Richards equation such that we may obtain the linearized Richards equation. We adopt the multiquadric function as the RBF in the meshless method for solving the linearized Richards equation. For simulating the unsaturated flow problems in layered heterogeneous soils, the flux and the head must satisfy the continuity condition at the interface. Numerical implementations including one- and two-dimensional unsaturated flow problems are established to validate the proposed method. Several application examples are carried out for modeling the two-dimensional hydrological process in multi-layered unsaturated soils.

The organization of the article is as follows. Section 2 introduces the mathematical formulation of unsaturated soil problems. We also demonstrate the numerical method to describe the numerical approximations to the linearized Richards equation for unsaturated flow problems in Section 2. In Section 3 and Section 4, numerical implementations and applications of unsaturated infiltration problems in homogeneous and heterogeneous porous media are conducted to give the accuracy, convergence and stability of the proposed meshless method. Findings are finally concluded in Section 5.

\section{The meshless method for modeling unsaturated flow}

\subsection{Formulation of Linearized Richards equation}

Based on the continuous equation and Darcy-Buckingham law, the nonlinear hydrological process in unsaturated soils is governed by the Richards equation, which can be expressed as

$$
\begin{aligned}
c(\Psi) \frac{\partial \Psi}{\partial t}= & \frac{\partial}{\partial x}\left(k_{x}(\Psi) \frac{\partial \Psi}{\partial x}\right)+\frac{\partial}{\partial y}\left(k_{y}(\Psi) \frac{\partial \Psi}{\partial y}\right) \\
& +\frac{\partial}{\partial z}\left(k_{z}(\Psi) \frac{\partial \Psi}{\partial z}\right)+\frac{\partial k_{z}(\Psi)}{\partial z}
\end{aligned}
$$

where $\Psi$ is the pressure head, $t$ is the time, $x$ points down the ground surface, $y$ points to the tangent of the topographic contour passing through the origin, $z$ is the vertical coordinate, $c(\Psi)$ is the specific moisture capacity function defined by $c(\Psi)=$ $\partial \theta(\Psi) / \partial \Psi, \theta(\Psi)$ is the moisture content, and $k_{x}(\Psi)$, $k_{y}(\Psi)$, and $k_{z}(\Psi)$ are unsaturated permeability functions in three dimensions, respectively.

Eq. (1) is the unsaturated flow governing equation in three dimensions for modeling the process of flow movement. Since the unsaturated permeability and the specific moisture capacity functions are functions of the pressure head, the above equation is highly nonlinear. If we consider the unsaturated porous media to be isotropic and homogeneous, eq. (1) can be rewritten as follows.

$$
\frac{\partial \theta(\Psi)}{\partial t}=\frac{\partial}{\partial x}\left(k(\Psi) \frac{\partial \Psi}{\partial x}\right)+\frac{\partial}{\partial z}\left(k(\Psi) \frac{\partial \Psi}{\partial z}\right)+\frac{\partial k(\Psi)}{\partial z} .
$$

Eq. (2) is the Richards equation governing two-dimensional flow in unsaturated porous media. We may normalize the permeability of unsaturated porous media and obtain the following equation.

$k(\Psi)=k_{r}(\Psi) k_{s}$,

where $k_{s}$ denotes the saturated permeability, and $k_{r}$ denotes the relative permeability. Substituting eq. (3) into eq. (2), we may find the following equation. 
$\frac{1}{k_{s}} \frac{\partial \theta(\Psi)}{\partial t}=\frac{\partial}{\partial x}\left(k_{r}(\Psi) \frac{\partial \Psi}{\partial x}\right)+\frac{\partial}{\partial z}\left(k_{r}(\Psi) \frac{\partial \Psi}{\partial z}\right)+\frac{\partial k_{r}(\Psi)}{\partial z}$.

To deal with the above nonlinear Richards equation, the characteristic functions including the characteristic curve of soil and water, the function of specific moisture capacity, and the unsaturated permeability function described the physical property of unsaturated porous media may be required.

One of the frequently used characteristic models to describe the physical property of unsaturated porous media is the exponential model proposed by Gardner, which can be expressed as follows [10].

$k_{r}(\Psi)=e^{\alpha \Psi}$,

$\theta(\Psi)=\left(\theta_{s}-\theta_{r}\right) e^{\alpha \Psi}+\theta_{r}$,

where $\alpha$ represents the pore size distribution parameter of unsaturated porous media, $\theta_{r}$ denotes the residual moisture content, and $\theta_{s}$ is the saturated moisture content.

Substituting eqs. (5) and (6) into eq. (4), the linearized Richards equation in two dimensions is obtained.

$\frac{\alpha\left(\theta_{s}-\theta_{r}\right)}{k_{s}} \frac{\partial \widehat{\Psi}}{\partial t}=\frac{\partial^{2} \widehat{\Psi}}{\partial x^{2}}+\frac{\partial^{2} \widehat{\Psi}}{\partial z^{2}}+\alpha \frac{\partial \widehat{\Psi}}{\partial z}$.

In the preceding equations, $\widehat{\Psi}=e^{\alpha \Psi}-e^{\alpha \Psi}$ which represents the linearized pressure head and $\Psi_{d}$ is the linearized pressure head while the soil is in dry condition. As a result, eq. (7) can be written as follows.

$\frac{\partial \widehat{\Psi}}{\partial t}=k_{\alpha} \frac{\partial^{2} \widehat{\Psi}}{\partial x^{2}}+k_{\alpha} \frac{\partial^{2} \widehat{\Psi}}{\partial z^{2}}+k_{\theta} \frac{\partial \widehat{\Psi}}{\partial z}$

$0=k_{\alpha} \frac{\partial^{2} \widehat{\Psi}}{\partial x^{2}}+k_{\alpha} \frac{\partial^{2} \widehat{\Psi}}{\partial z^{2}}+k_{\theta} \frac{\partial \widehat{\Psi}}{\partial z}$,

where $k_{\theta}=\frac{k_{s}}{\alpha\left(\theta_{s}-\theta_{r}\right)}$, and $k_{\alpha}=\frac{k_{\theta}}{\alpha}$. Eqs. (8) and (9) are the two-dimensional transient and steady-state linearized Richards equations, respectively.

\subsection{The meshless method with the RBF}

In this section, the meshless method with the RBF for solving unsaturated flow in heterogeneous soils is introduced. We consider a $d$-dimensional domain, $\Omega \subset \mathrm{R}^{d}$, enclosed by the boundary, $\partial \Omega$. The equation in the unsaturated soil can be written as follows.

$D \Psi(\mathbf{x})=f(\mathbf{x})$ in $\Omega$,
$\Psi(\mathbf{x})=g(\mathbf{x})$ on $\partial \Omega$,

where $D$ denotes a differential operator, $\mathbf{x}=(x, z)$, $f(\mathbf{x})$ represents the function value, and $g(\mathbf{x})$ represents the boundary value. We assume $N$ to be the point number in $\Omega$. The meshless method with the $\mathrm{RBF}$ is applied by assuming the following equation.

$\widehat{\Psi}(\mathbf{x})=\sum_{j=1}^{N} \lambda_{j} \phi_{j}(\mathbf{x})$,

where $\phi_{j}(\mathbf{x})$ represents the $\mathrm{RBF}$, and $\lambda_{j}$ represents unknown coefficients to be determined. The RBF approximation at the $i^{\text {th }}$ collocation point, $\widehat{\Psi}\left(\mathbf{x}_{i}\right)$, may be expressed as follows.

$\widehat{\Psi}\left(\mathbf{x}_{i}\right)=\sum_{j=1}^{N} \lambda_{j} \phi_{j}\left(\mathbf{x}_{j}\right)$,

where $\mathbf{x}_{j}$ represents an arbitrary point. The $N$ points with coordinates $\mathbf{x}_{1}, \ldots, \mathbf{x}_{N}$ are collocated in the computational domain. In the preceding equation, we consider the multiquadratic RBF, which is expressed as follows.

$\phi_{j}\left(\mathbf{x}_{j}\right)=\sqrt{(r \epsilon)^{2}+1}$,

where $r$ represents the radius, $r=\left|\mathbf{x}_{i}-\mathbf{x}_{j}\right|$, and $\epsilon$ represents the shape parameter. It is found that some common RBFs in the infinitely differentiable class depend on a shape parameter, $\epsilon$. The shape parameter is a key factor in adopting multiquadratic approximation in the RBF method, which may affect the accuracy of the numerical solutions significantly [27]. To determine the shape parameter, the adaptive residual subsampling algorithm proposed by Driscoll and Heryudon is adopted in this study to prevent the increase of the condition number of the matrix [7].

Consequently, the following equations can be obtained by collocating with the above equations at the inner points and the boundary values at the boundary points.

$$
\begin{aligned}
& D \widehat{\Psi}\left(\mathbf{x}_{i}\right)=\sum_{j=1}^{N} \lambda_{j} D \phi_{j}\left(\mathbf{x}_{j}\right)=f\left(\mathbf{x}_{i}\right), i=1, \ldots, N_{I}, \\
& j=1, \ldots, N \\
& \widehat{\Psi}\left(\mathbf{x}_{i}\right)=\sum_{j=1}^{N} \lambda_{j} \phi_{j}\left(\mathbf{x}_{j}\right)=g\left(\mathbf{x}_{i}\right), i=N_{I}+1, \ldots, N, \\
& \quad j=1, \ldots, N .
\end{aligned}
$$


In the preceding equations, $N_{I}$ is the inner point number. Substituting eq. (13) into eq. (16), we may obtain the following equation.

$\widehat{\Psi}\left(\mathbf{x}_{i}\right)=\sum_{j=1}^{N} \lambda_{j} \phi_{j}\left(\mathbf{x}_{j}\right)=\sum_{j=1}^{N} \lambda_{j} \sqrt{\left(r_{i, j} \epsilon\right)^{2}+1}$.

We consider the second derivative to $x$ of eq. (17) as following equation. matrix. The above equation can be rewritten as follows.

$\left[\begin{array}{l}\mathbf{B I} \\ \mathbf{B B}\end{array}\right][\lambda]=\left[\begin{array}{l}\mathbf{F I} \\ \text { FB }\end{array}\right]$.

In the preceding equations, BI represents the $N_{I} \times N$ submatrix from the inner collocation points, BB represents the $N_{B} \times N$ submatrix from the boundary collocation points, FI denotes the function

$\frac{\partial^{2} \widehat{\psi}}{\partial x^{2}}=\frac{\partial^{2}}{\partial x^{2}} \sum_{j=1}^{N} \lambda_{j} \varphi_{j}\left(\mathbf{x}_{j}\right)=\sum_{j=1}^{N} \lambda_{j} \frac{\partial^{2} \sqrt{\left(r_{i, j} \epsilon\right)^{2}+1}}{\partial x^{2}}=\sum_{j=1}^{N} \lambda_{j} \frac{\epsilon^{2}}{\left[\left(r_{i, j} \epsilon\right)^{2}+1\right]^{3 / 2}}$.

We further consider the first derivative to $z$ of eq. (17) as following equation.

$$
\begin{aligned}
\frac{\partial \widehat{\Psi}}{\partial z}=\frac{\partial}{\partial z} \sum_{j=1}^{N} \lambda_{j} \phi_{j}\left(\mathbf{x}_{j}\right) & =\sum_{j=1}^{N} \lambda_{j} \frac{\partial \sqrt{\left(r_{i, j} \epsilon\right)^{2}+1}}{\partial z} \\
& =\sum_{j=1}^{N} \lambda_{j} \frac{\epsilon^{2}\left(z_{i}-z_{j}\right)}{\sqrt{\left(r_{i, j} \epsilon\right)^{2}+1}} .
\end{aligned}
$$

Substituting above equations into eq. (9), we may obtain the following equation.

$$
\begin{aligned}
& k_{\alpha} \sum_{j=1}^{N} \lambda_{j} \frac{r_{i, j}^{2} \epsilon^{4}+2 \epsilon^{2}}{\left[\left(r_{i, j} \epsilon\right)^{2}+1\right]^{3 / 2}} \\
& +k_{\theta} \sum_{j=1}^{N} \lambda_{j} \frac{\epsilon^{2}\left(z_{i}-z_{j}\right)}{\sqrt{\left(r_{i, j} \epsilon\right)^{2}+1}}=0 .
\end{aligned}
$$

Finally, we collocate with the inner and boundary points by using eqs. (15) and (16). To determine the unknown coefficient, we impose the approximate solution to satisfy the linearized Richards equation with the boundary conditions at any collocation points. We then achieve the following system of linear equations.

$$
\left[\begin{array}{l}
D \phi_{j}\left(\mathbf{x}_{j}\right) \\
\phi_{j}\left(\mathbf{x}_{j}\right)
\end{array}\right]\left[\lambda_{j}\right]=\left[\begin{array}{l}
f\left(\mathbf{x}_{i}\right) \\
g\left(\mathbf{x}_{i}\right)
\end{array}\right] \text {. }
$$

For simplicity, the above system of linear equation can be written as

$\mathbf{B} \boldsymbol{\lambda}=\mathbf{F}$,

where $\lambda$ is the unknown coefficients to be evaluated, which can be expressed as $\lambda=\left[\lambda_{1}, \ldots, \lambda_{N}\right]^{\mathrm{T}}, \mathbf{F}$ is the known functions with the size of $N \times 1, \mathbf{B}$ is a $N \times N$ values at the inner points which is a $N_{I} \times 1$ vector, FB denotes the function values at the boundary points which is a $N_{B} \times 1$ vector, $N_{B}$ is the of boundary point number, and $N$ represents the total number of points which can be expressed as $N=$ $N_{I}+N_{B}$. Therefore, the above equation can be rewritten as

$$
\left[\begin{array}{llll}
B I_{1,1} & B I_{1,2} & \cdots & B I_{1, N} \\
\vdots & \vdots & \cdots & \vdots \\
B I_{N_{I}, 1} & B I_{N_{I}, 2} & \vdots & B I_{N_{I}, N} \\
B B_{1,1} & B B_{1,2} & \ddots & B B_{1, N} \\
\vdots & \vdots & \cdots & \vdots \\
B B_{N_{B}, 1} & B B_{N_{B}, 2} & \cdots & B B_{N_{B}, N}
\end{array}\right]\left[\begin{array}{l}
\lambda_{1} \\
\vdots \\
\lambda_{N_{I}} \\
\lambda_{N_{I}+1} \\
\vdots \\
\lambda_{N}
\end{array}\right]=\left[\begin{array}{l}
F I_{1} \\
\vdots \\
F I_{N_{I}} \\
F B_{N_{I}+1} \\
\vdots \\
F B_{N}
\end{array}\right],
$$

where $F I_{i}$ denotes the function values at the inner points which is a $N_{I} \times 1$ vector, $F B_{i}$ denotes the function values at the boundary points which is a $N_{B} \times 1$ vector, $B I_{i, j}$ is a $N_{I} \times N$ matrix, $B B_{i, j}$ denotes a $N_{B} \times N$ matrix and $\lambda_{1}, \ldots, \lambda_{N}$ denote unknowns. In the preceding equations, $B I_{i, j}$ and $B B_{i, j}$ may be found using the following equation.

$$
\begin{aligned}
B I_{i, j} & =k_{\alpha} \frac{r_{i, j}^{2} \epsilon^{4}+2 \epsilon^{2}}{\left[\left(r_{i, j} \epsilon\right)^{2}+1\right]^{3 / 2}}+k_{\theta} \frac{\epsilon^{2}\left(z_{i}-z_{j}\right)}{\sqrt{\left(r_{i, j} \epsilon\right)^{2}+1}}, i=1, \ldots, N_{I} \text { and } \\
& =1, \ldots, N,
\end{aligned}
$$

$B B_{i, j}=\sqrt{\left(r_{i, j} \epsilon\right)^{2}+1}, i=1, \ldots, N_{B}$ and $j=1, \ldots, N$.

The unknowns for the two-dimensional problem may be found for solving eq. (24). To compute the unsaturated pressure head field for the two-dimensional problem, the inner points must be collocated. The unsaturated pressure head field at inner points may be computed using eq. (24). 


\subsection{The RBF method for modeling flow in heterogeneous soils}

Since the appearance of layered heterogeneous soils is usually easily found than homogeneous soils in practice, we need to tackle the flow in heterogeneous soils. For the analysis of flow in multi-layered porous media, the domain decomposition method (DDM) is utilized [36].

The DDM is commonly used to solve the problem with different physical characteristics in each subdomain. We first split the domain into two subdomains which are intersected only at the interface. Hence, each subdomain is regard as an independent soil layer with its own permeability. The boundary and inner collocation points are placed in each subdomain. At the interface, the flux and the head must satisfy the continuity condition.

In this article, we consider a rectangular domain, $\Omega$, which can be split into two intersected

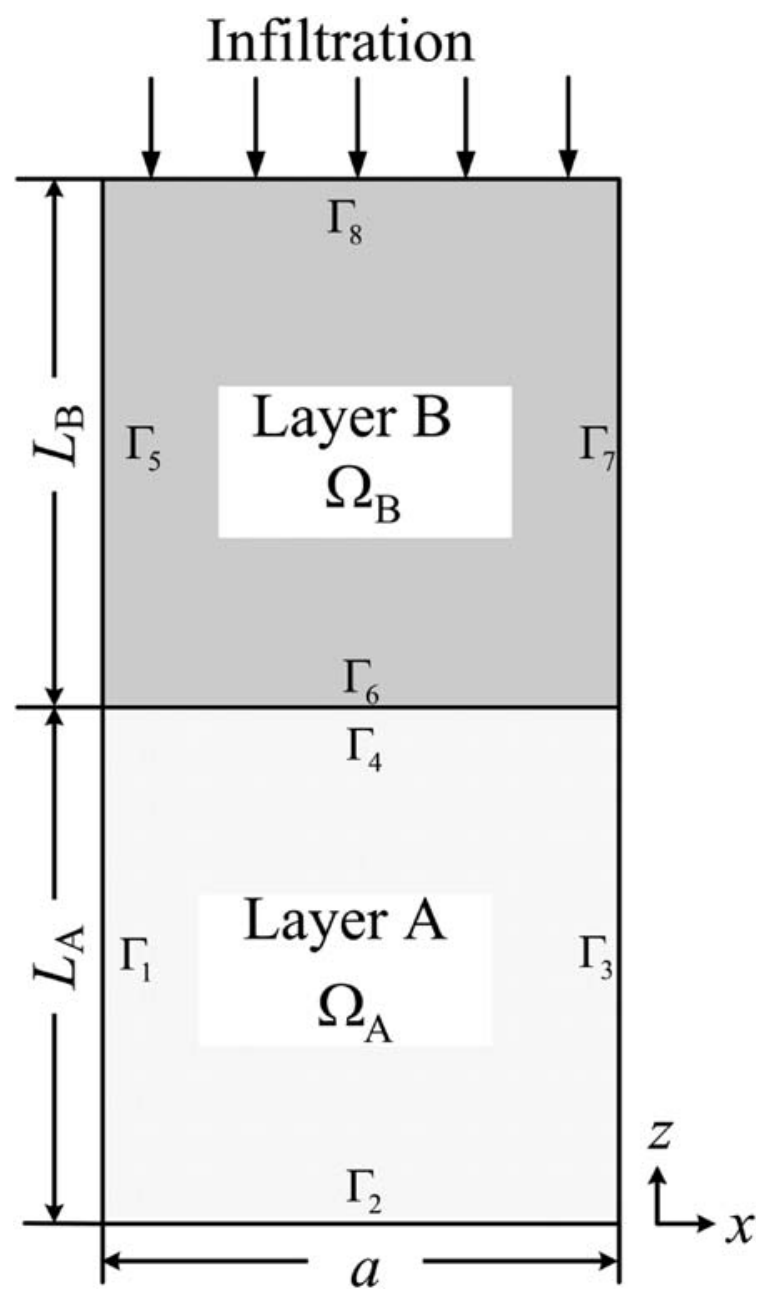

Fig. 1. Illustration of two-dimensional infiltration problem in unsaturated two-layered porous media. subdomains, $\Omega_{\mathrm{A}}$ and $\Omega_{\mathrm{B}}$, as depicted in Fig. 1. To simulate the unsaturated flow in heterogeneous soils, the rectangular domain is divided into $\Gamma_{1}, \Gamma_{2}$, $\Gamma_{3}, \ldots, \Gamma_{8}$. At $\Omega_{\mathrm{A}}$ subdomains, the sub-boundaries include $\Gamma_{1}, \Gamma_{2}, \Gamma_{3}$ and $\Gamma_{4}$; At $\Omega_{\mathrm{B}}$ subdomains, the sub-boundaries include $\Gamma_{5}, \Gamma_{6}, \Gamma_{7}$ and $\Gamma_{8}$. At the interface, the sub-boundaries, $\Gamma_{4}$ and $\Gamma_{6}$, are overlapped at the same position. Therefore, additional boundary conditions are imposed on the boundary points to ensure the flux and the head at the interface must be equal.

$\left.\widehat{\Psi}\right|_{\Gamma_{4}}=\widehat{\Psi}||_{\Gamma_{6}}$,

$\left.\frac{\partial \widehat{\Psi}}{\partial n}\right|_{\Gamma_{4}}=\frac{\partial \widehat{\Psi}}{\partial n}||_{\Gamma_{6}}$.

Eq. (28) can be written as

$\frac{\partial \widehat{\Psi}(\mathbf{x})}{\partial n}=\frac{\partial \widehat{\Psi}(\mathbf{x})}{\partial x} n_{x}+\frac{\partial \widehat{\Psi}(\mathbf{x})}{z} n_{z}$.

where $n_{x}$ and $n_{z}$ are vectors in the normal direction for $x$ and $z$, respectively. Substituting eqs. (18) and (19) into eq. (29), we may find the following equation.

$\frac{\partial \widehat{\Psi}(\mathbf{x})}{\partial n}=\sum_{j=1}^{N} \lambda_{j} \frac{\epsilon^{2}\left(x_{i}-x_{j}\right)}{\sqrt{\left(r_{i, j} \epsilon\right)^{2}+1}} n_{x}+\sum_{j=1}^{N} \lambda_{j} \frac{\epsilon^{2}\left(z_{i}-z_{j}\right)}{\sqrt{\left(r_{i, j} \epsilon\right)^{2}+1}} n_{z}$.

\section{Validation examples}

\subsection{One-dimensional infiltration process in unsaturated homogeneous soils}

The first numerical example investigated is the analysis of a one-dimensional infiltration problem in the vadose zone [35]. The one-dimensional unsaturated flow in the homogeneous isotropic porous media is described by the Richards equation as

$0=\frac{\partial}{\partial z}\left(k_{r}(\Psi) \frac{\partial \Psi}{\partial z}\right)+\frac{\partial k_{r}(\Psi)}{\partial z}$.

The thickness of the soil is considered to be $L$. At the top side of the unsaturated soil which is the ground surface, the infiltration is remained by keeping the pressure head to zero. On the bottom side of the unsaturated soil, the boundary condition is assumed to be in dry condition, $\Psi_{d}$. Thus, the boundaries on the top and bottom sides of the unsaturated soil are considered to be Dirichlet boundary condition, which are as follows. 
$\Psi(z=L)=0$.

$\Psi(z=0)=\Psi_{d}$.

Eqs. (32) and (33) denote the top and bottom boundary conditions of the unsaturated soil, respectively. The above equations can be converted to linearized pressure head as following equations.

$\widehat{\Psi}(z=L)=1$.

$\widehat{\Psi}(z=0)=0$.

To describe the nonlinearity of relative permeability, the exponential model is adopted (Gardner, 1958). In this example, we assume the soil thickness is $10 \mathrm{~m}$, the soil type is sand, the pore size distribution parameter is $1.5 \times 10^{-2}, \Psi_{d}$ is $-100 \mathrm{~m}$, the saturated permeability to be $10^{-2} \mathrm{~m} / \mathrm{s}$, the saturated moisture content to be 0.5 , and the residual moisture content to be 0.1 .

The analytical solution of one-dimensional infiltration process in unsaturated homogeneous porous media can be obtained as follows [32].

$\widehat{\Psi}(z)=\left(1-e^{\alpha \Psi}\right) e^{\frac{\alpha}{2}(L-z)}\left[\frac{\sinh \left(\frac{\alpha z}{2}\right)}{\sinh \left(\frac{\alpha L}{2}\right)}\right]$.

In this study, only 2 boundary points and 8 inner points are collocated. The Dirichlet values are imposed on the top and bottom boundary points. The inner points are collocated in equal interval within the domain. The shape parameter for this

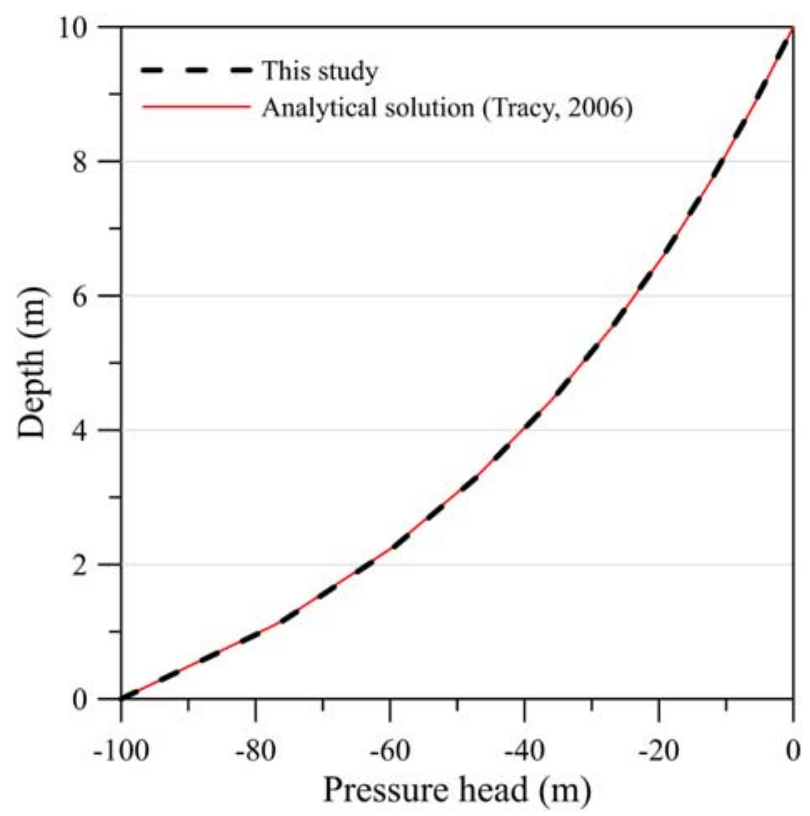

Fig. 2. Result comparison for an one-dimensional infiltration problem. analysis is 0.09 . Fig. 2 shows the results using our numerical method as well as the exact solution developed by Ref. [32]. The computed results agree very well with the exact solution. The validity of the method is achieved in one-dimensional unsaturated problem with only 10 collocation points.

\subsection{Two-dimensional infiltration process in unsaturated homogeneous porous media}

Considering a two-dimensional unsaturated flow in homogeneous soils, the flow in the isotropic and homogeneous soils is described by the Richards equation, which is expressed as follows.

$\frac{\partial}{\partial x}\left(k_{r}(\Psi) \frac{\partial \Psi}{\partial x}\right)+\frac{\partial}{\partial z}\left(k_{r}(\Psi) \frac{\partial \Psi}{\partial z}\right)+\frac{\partial k_{r}(\Psi)}{\partial z}=0$.

In this example, the length $a$ and the thickness $L$ are both set to $50 \mathrm{~m}$, as shown in Fig. 3 (a). At the top side of the unsaturated soil which is the ground surface, the zero pressure head is at the central and rapidly decreasing to negative pressure head, i.e. dry conditions at both sides, as depicted in Fig. 3 (a). On the left, bottom and right sides of the domain, the boundary condition is assumed to be in dry condition, $\Psi_{d}$. Thus, the equations of boundary values for the domain can be written as follows.

$\Psi(x, z=L)=\frac{1}{\alpha} \ln \left(e^{\alpha \Psi_{d}}+\left(1-e^{\alpha \Psi_{d}}\right)\right.$
$\left.\times\left(\frac{3}{4} \sin \left(\frac{\pi x}{a}\right)-\frac{1}{4} \sin \left(\frac{3 \pi x}{a}\right)\right)\right)$,

$\Psi(x, z=0)=\Psi_{d}$,

$\Psi(x=0, z)=\Psi_{d}$,

$\Psi(x=a, z)=\Psi_{d}$.

Eqs. (38) - (41) denote the top, bottom, left as well as right boundary conditions of the two-dimensional unsaturated soil, respectively. The analytical solution of two-dimensional infiltration process in unsaturated homogeneous porous media can be obtained as follows [32].

$$
\begin{aligned}
\widehat{\Psi}(x, z)= & \left(1-e^{\alpha \Psi_{d}}\right) e^{\frac{\alpha(L-z)}{2}}\left(\frac{3}{4} \sin \left(\frac{\pi x}{a}\right) \frac{\sinh \left(\beta_{1} z\right)}{\sinh \left(\beta_{1} L\right)}\right. \\
& \left.-\frac{1}{4} \sin \left(\frac{3 \pi x}{a}\right) \frac{\sinh \left(\beta_{3} z\right)}{\sinh \left(\beta_{3} L\right)}\right),
\end{aligned}
$$

where $\beta_{1}=\sqrt{\frac{\alpha_{g}^{2}}{4}+\left(\frac{\pi}{a}\right)^{2}}$ and $\beta_{3}=\sqrt{\frac{\alpha_{g}^{2}}{4}+\left(\frac{3 \pi}{a}\right)^{2}}$.

To describe the nonlinearity of relative hydraulic conductivity, the Gardner exponential model is also 

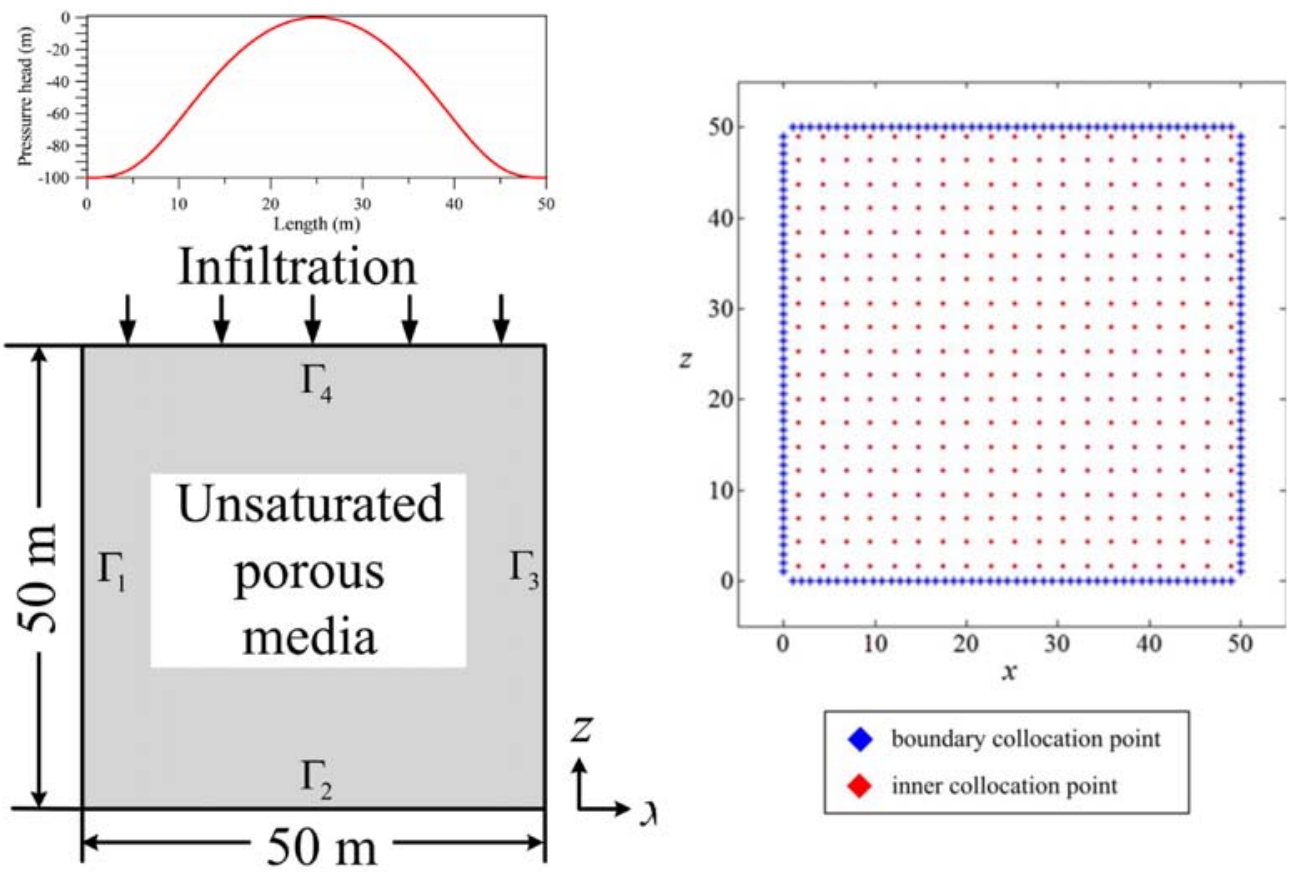

(a) Illustration of infiltration problem in two dimensions.

(b) Points collocated on the boundary and within the domain.

Fig. 3. Illustration of example 3.2.

adopted. In this example, we assume the soil type to be sand, the pore size distribution parameter to be $1.5 \times 10^{-2}, \Psi_{d}$ to be $-100 \mathrm{~m}$, the saturated permeability to be $10^{-2} \mathrm{~m} / \mathrm{s}$, the saturated moisture content to be 0.5 , and the residual moisture content to

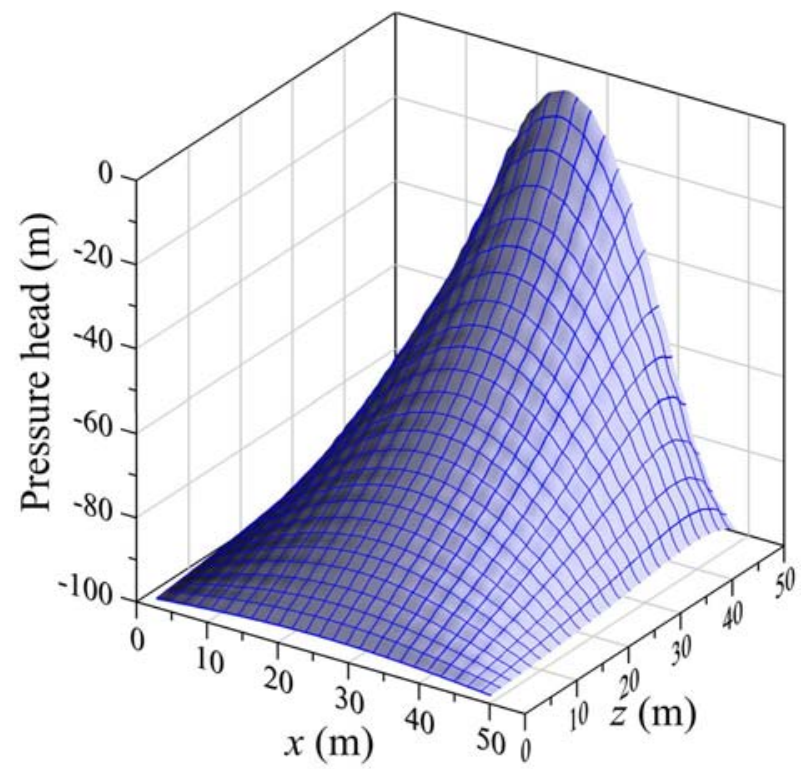

Fig. 4. The computed results for the two-dimensional infiltration problem in unsaturated homogeneous porous media. be 0.1 .200 boundary points and 361 inner points are collocated, as displayed in Fig. 3 (b). The shape parameter for the analysis is 0.034 . Fig. 4 shows the results of our proposed method. Fig. 5 presents the relative error of two-dimensional infiltration problem in unsaturated homogeneous porous media. It

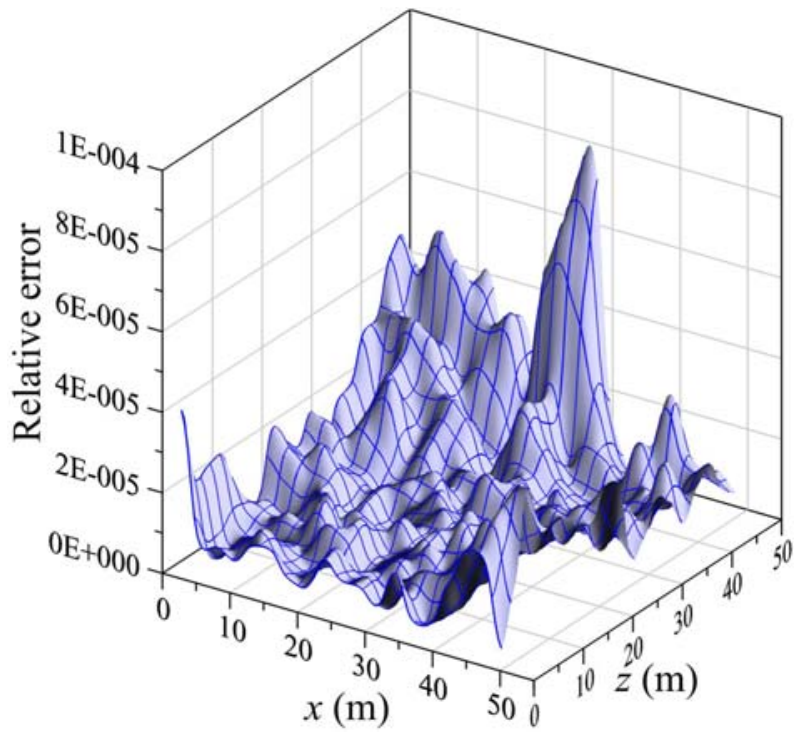

Fig. 5. The relative error of the numerical results for the infiltration problem in two dimensions. 
is indicated that the accuracy of our proposed method can reach up to the order of $10^{-5}$.

To examine the robust of the proposed approach, we consider that the boundary data are contaminated by the random noise. The noised data on accessible boundary can be represented as follows.

$\tilde{\Psi}(x, z)=\Psi(x, z) \times(1+s \times$ rand $)$,

where $\Psi(x, z)$ is the exact boundary data, $\tilde{\Psi}(x, z)$ is the noised data on accessible boundary, rand is the random number generated by the uniform distribution in the range of $[-1,1]$, and $s$ is the noise level. The uniform probability density function has been considered to generate noised data on accessible
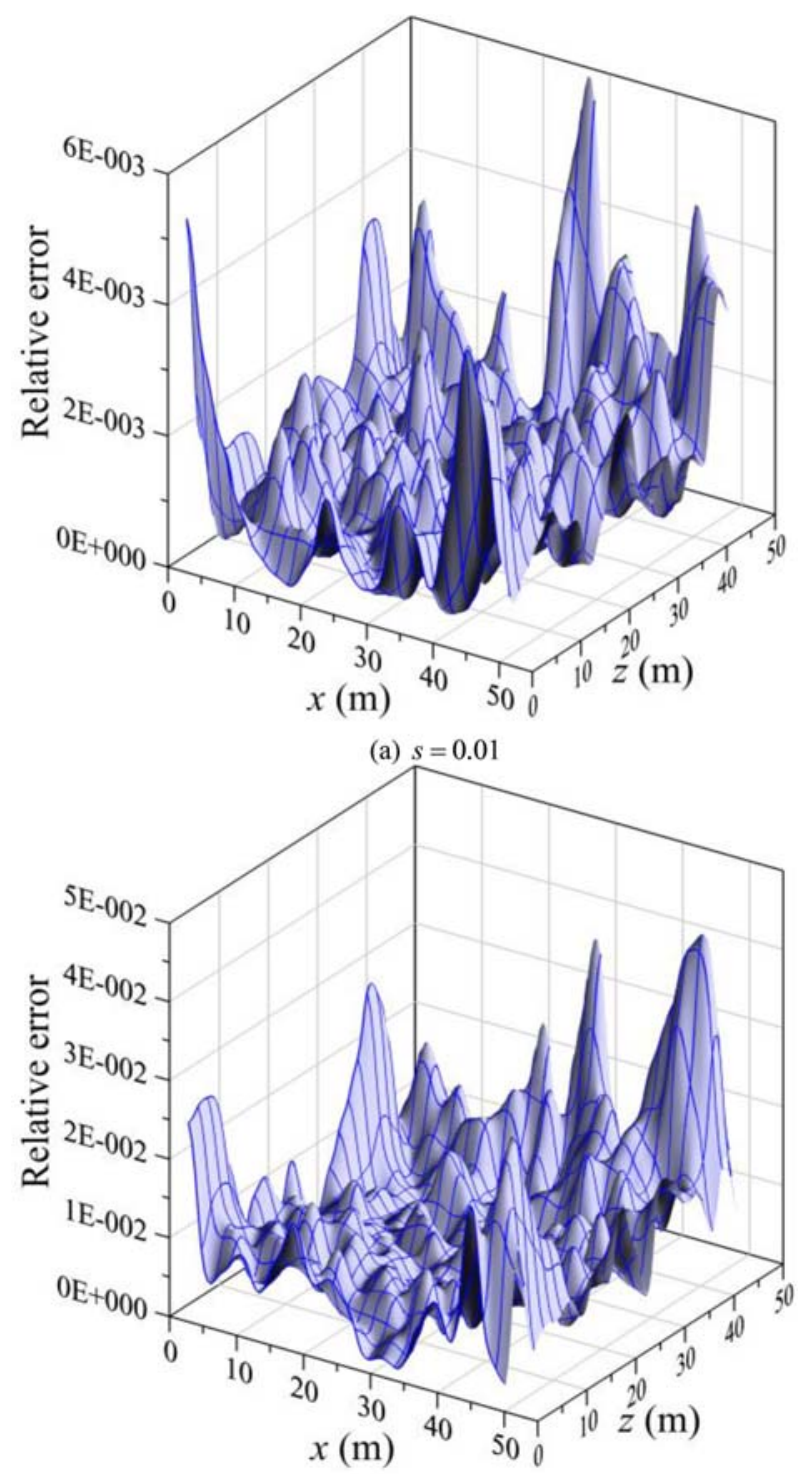

(b) $s=0.05$

Fig. 6. The relative error of the numerical results for the infiltration problem in two dimensions. boundaries. In this example, two different noise levels with $s=0.01$, and $s=0.05$ are considered.

The maximum relative error for two-dimensional unsaturated flow problems is illustrated in Fig. 6. It is found that the maximum relative error may reach to the order of $6.85 \times 10^{-3}$ and $1.17 \times 10^{-2}$ for the noise level $s=0.01$, and $s=0.05$, respectively, as shown in Fig. 6 (a), (b).

\section{Application examples}

\subsection{Two-dimensional infiltration process in unsaturated two-layered soils}

The first application example under consideration is the analysis of the infiltration problems in an unsaturated two-layered soil. Fig. 7 (a) depicts the profile of the unsaturated two-layered porous media for this example. The length and the height of the soil are both considered to be $10 \mathrm{~m}$. Both layer A as well as layer $B$ have the thickness of $5 \mathrm{~m}$, as shown in Fig. 7 (a) in which layer A has larger permeability than that of layer B. The soil type for the top soil (layer B) and the bottom soil (layer A) are assumed to be silty loam and sand, respectively. The saturated permeability in layer A and layer B are $10^{-3}$ and $10^{-4} \mathrm{~m} / \mathrm{s}$, respectively. To describe the nonlinearity of relative permeability, the Gardner exponential model is adopted. The unsaturated soil parameters including the pore size distribution parameter, the saturated and residual moisture contents for two soil layers are shown in Table 1.

The boundary conditions including $\Gamma_{1}, \Gamma_{2}, \ldots, \Gamma_{8}$ are shown in Fig. 7 (a). On the top side of the unsaturated porous media, there is infiltration on the ground surface where the pressure head set to zero.

$\widehat{\Psi}(x, z=L)=\left(1-e^{\alpha \Psi_{d}}\right)$ on $\Gamma_{8}$.

On the bottom, the boundary is in dry condition, $\Psi_{d}=-1000 \mathrm{~m}$, where the Dirichlet value is given as

$\widehat{\Psi}(x, z=0)=0$ on $\Gamma_{2}$,

The left and right sides are the Neumann boundaries which can be written as follows.

$\frac{\partial \widehat{\Psi}(x=0, z)}{\partial z}=0$ on $\Gamma_{1}$ and $\Gamma_{5}$,

$\frac{\partial \widehat{\Psi}(x=a, z)}{\partial z}=0$ on $\Gamma_{3}$ and $\Gamma_{7}$.

For the infiltration problems in an unsaturated two-layered soil, we have to consider the discontinuities in the parameters that characterize the unsaturated soil layers. At the interface between soil 


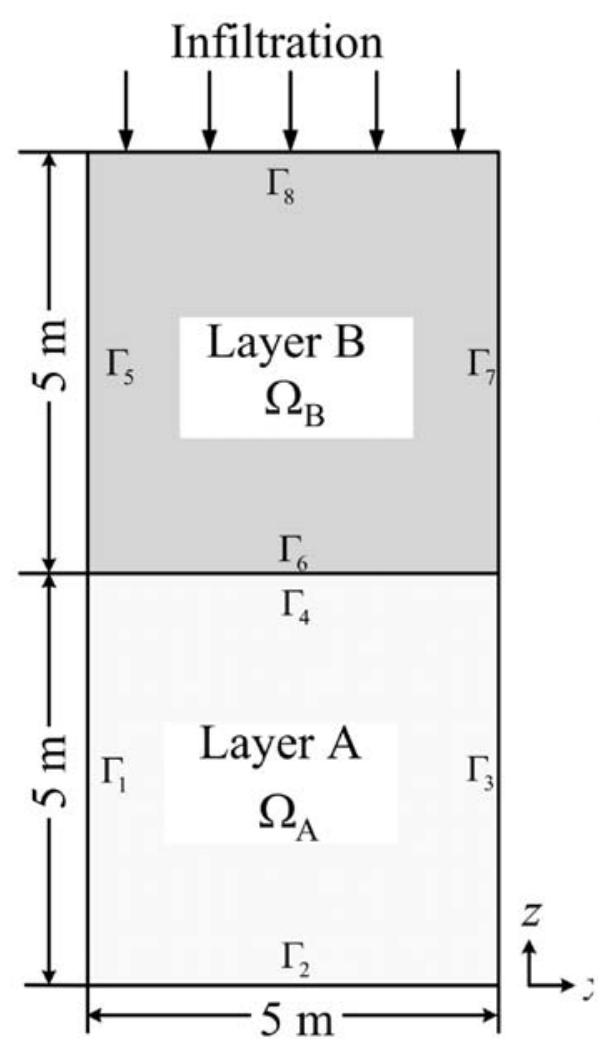

(a) Illustration of the infiltration problem in two dimensions.

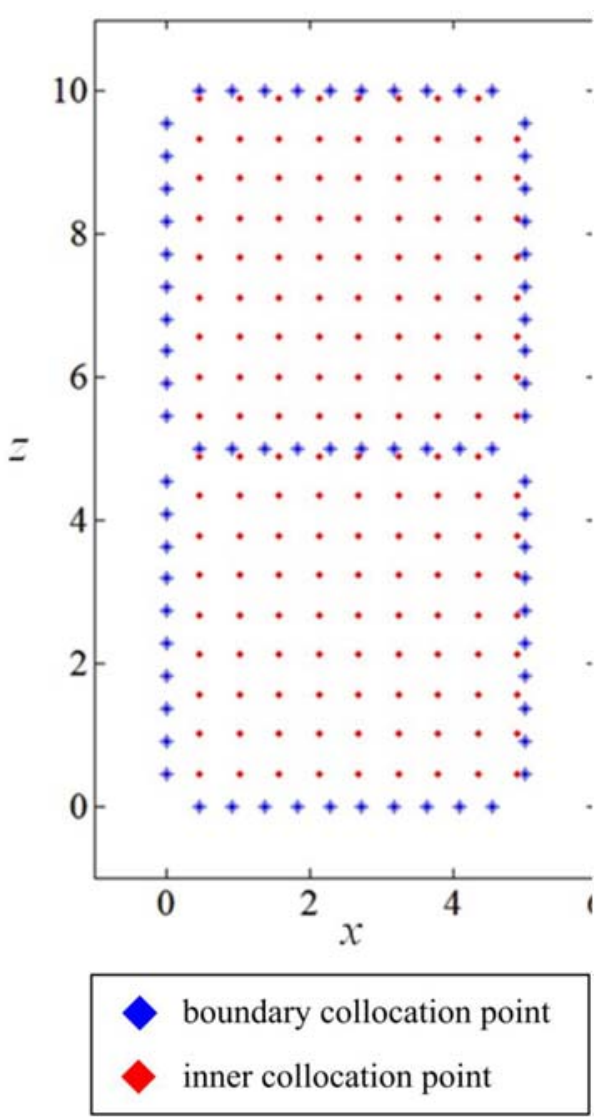

(b) Points collocated on the boundary and within the domain.

Fig. 7. Illustration of example 4.1.

layers, the continuity of flux and the Darcy-Buckingham law are required. The boundary conditions are expressed as following equations.

$\left.\widehat{\Psi}\right|_{\Gamma_{4}}=\left.\widehat{\Psi}\right|_{\Gamma_{6}}$ at $\Gamma_{4}$ and $\Gamma_{6}$.

$\left.\frac{\partial \widehat{\Psi}}{\partial n}\right|_{\Gamma_{4}}=\left.\frac{\partial \widehat{\Psi}}{\partial n}\right|_{\Gamma_{6}}$ at $\Gamma_{4}$ and $\Gamma_{6}$.

In this example, the shape parameter is $2.4 \times$ $10^{-3}$. The boundary and inner points are 80 and 162, respectively, as depicted in Fig. 7 (b). The profile of the pressure head in a two-layered unsaturated soil is depicted in Fig. 8. Since there is

Table 1. Unsaturated soil parameters.

\begin{tabular}{lll}
\hline layer & layer A & layer B \\
\hline soil type & sand & silty loam \\
pore size distribution parameter $(1 / \mathrm{m})$ & $4 \times 10^{-3}$ & $8 \times 10^{-3}$ \\
hydraulic conductivity $(\mathrm{m} / \mathrm{s})$ & $10^{-3}$ & $10^{-4}$ \\
saturated moisture content & 0.50 & 0.32 \\
residual moisture content & 0.11 & 0.03 \\
\hline
\end{tabular}

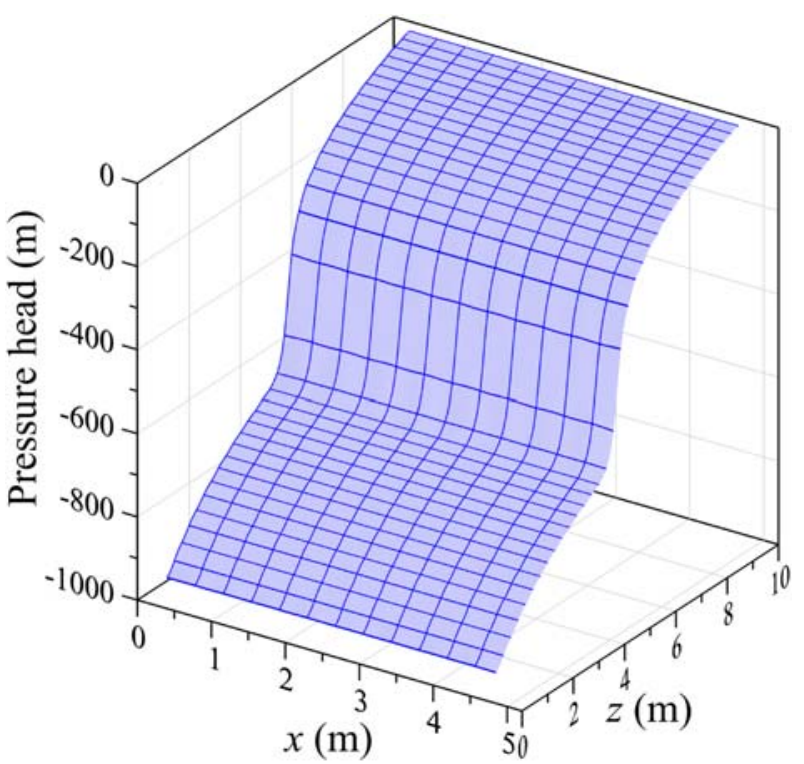

Fig. 8. The computed pressure head for the two-dimensional infiltration problem. 


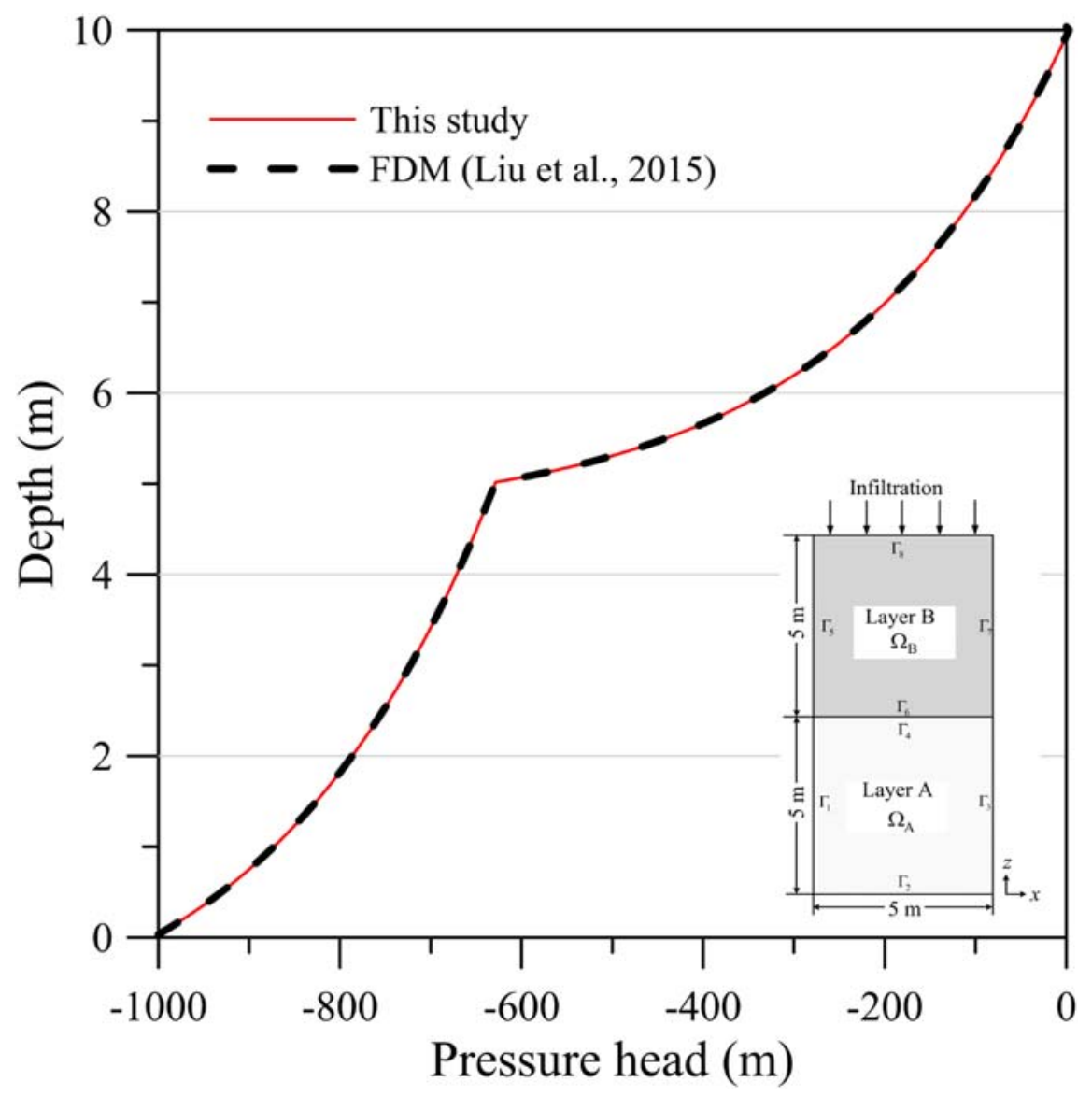

Fig. 9. The pressure head profiles for the two-dimensional infiltration problem.

infiltration on the ground level remaining the constant pressure head, the hydrological process may continue infiltrating to the unsaturated two-layered soil, as shown in Fig. 9. It is found that the infiltration starts in the less conductive layer (layer B). When the infiltration from the ground surface reach the interface with the high conductive soil (layer A), the amount of unsaturated flows to layer A may be dissipated easily due to the high conductivity of the layer A [28].

Liu et al. have applied the FDM to solve this infiltration problem. We therefore compare the computed results with that of the FDM, as depicted in Fig. 9. The results agree very well with those of the FDM [20].

\subsection{Two-dimensional infiltration process in an unsaturated three-layered soil}

The profile of the two-dimensional unsaturated three-layered soil including layer A, layer B, and layer $C$ for this example is shown in Fig. 10 (a). The length and the height of the soil are considered to be 5 and $10 \mathrm{~m}$, respectively. The thickness of layer $\mathrm{A}$, layer $B$, and layer $C$ are considered to be $4,2,4 \mathrm{~m}$, as shown in Fig. 10 (a).

In this example, the soil type of layer A and layer $\mathrm{C}$ are considered to be sand, where the saturated permeability in layer $A$ and layer $C$ are $10^{-3} \mathrm{~m} / \mathrm{s}$. The soil type of layer $B$ is considered to be silty loam, where the saturated permeability in layer B is $10^{-9} \mathrm{~m} / \mathrm{s}$. Other soil parameters including the pore size distribution parameter, the saturated moisture content, and the residual moisture content for two soil layers adopted for this example are listed in Table 2.

The boundary conditions including $\Gamma_{1}, \Gamma_{2}, \ldots, \Gamma_{12}$ are shown in Fig. 10 (a). On the top side of the unsaturated porous media, the infiltration is maintained on the ground surface where the pressure head set to zero. On the bottom, the boundary is assumed to be dry condition, $\Psi_{d}=-1000 \mathrm{~m}$. The right and left boundaries are considered to be no-flow Neumann boundary condition. 


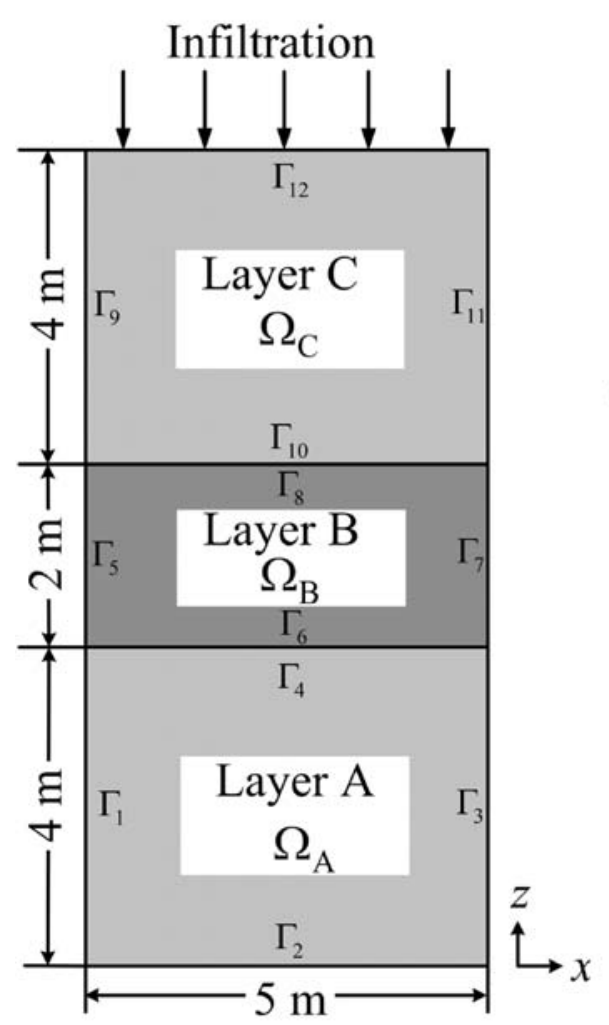

(a) Illustration of the infiltration problem in two dimensions.

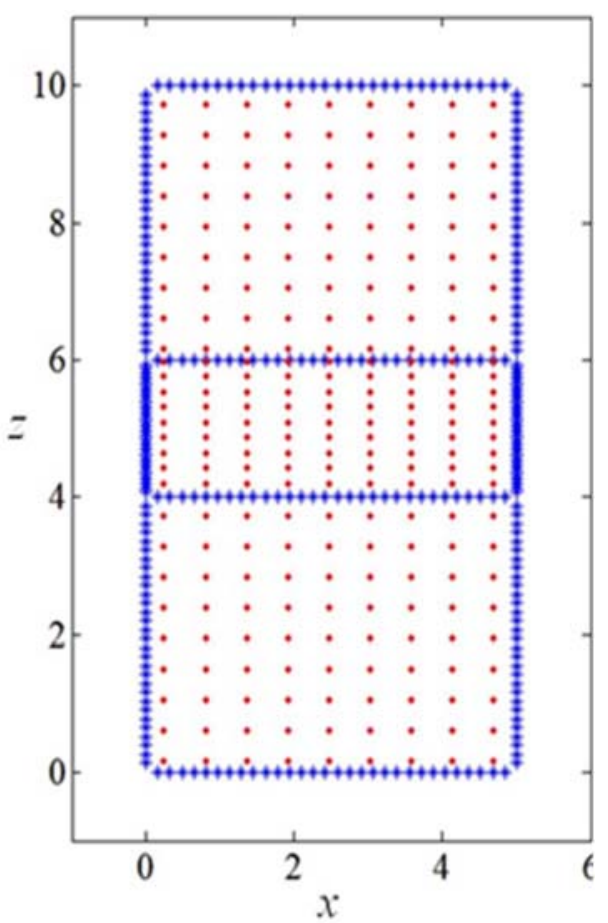

boundary collocation point inner collocation point

(b) Points collocated on the boundary and within the domain.

Fig. 10. Illustration of example 4.2.

The Dirichlet boundary conditions at the top side of the unsaturated soil, $\Gamma_{12}$, and the bottom side of the unsaturated soil, $\Gamma_{2}$, are given as following equations.

$\widehat{\Psi}(x, z=L)=\left(1-e^{\alpha \Psi_{d}}\right)$ on $\Gamma_{12}$,

$\widehat{\Psi}(x, z=0)=0$ on $\Gamma_{2}$.

At $\Gamma_{1}, \Gamma_{3}, \Gamma_{5}, \Gamma_{7}, \Gamma_{9}$ and $\Gamma_{11}$, the Neumann boundaries are given as follows.

$\frac{\partial \widehat{\Psi}(x=0, z)}{\partial z}=0$ on $\Gamma_{1}, \Gamma_{5}$ and $\Gamma_{9}$,

Table 2. Unsaturated soil parameters.

\begin{tabular}{|c|c|c|c|}
\hline layer & layer $\mathrm{A}$ & layer B & layer C \\
\hline soil type & sand & clay & sand \\
\hline $\begin{array}{l}\text { pore size distribution } \\
\text { parameter }(1 / \mathrm{m})\end{array}$ & $4 \times 10^{-3}$ & $8 \times 10^{-3}$ & $4 \times 10^{-3}$ \\
\hline Permeability $(\mathrm{m} / \mathrm{s})$ & $10^{-3}$ & $10^{-9}$ & $10^{-3}$ \\
\hline saturated moisture content & 0.50 & 0.32 & 0.50 \\
\hline residual moisture content & 0.11 & 0.03 & 0.11 \\
\hline
\end{tabular}

$\frac{\partial \widehat{\Psi}(x=a, z)}{\partial z}=0$ on $\Gamma_{3}, \Gamma_{7}$ and $\Gamma_{11}$.

At two interfaces, the boundary conditions are expressed as following equations.

$\left.\widehat{\Psi}\right|_{\Gamma_{4}}=\left.\widehat{\Psi}\right|_{\Gamma_{6}},\left.\frac{\partial \widehat{\Psi}}{\partial n}\right|_{\Gamma_{4}}=\left.\frac{\partial \widehat{\Psi}}{\partial n}\right|_{\Gamma_{6}}$ at $\Gamma_{4}$ and $\Gamma_{6}$.

$\left.\widehat{\Psi}\right|_{\Gamma_{8}}=\left.\widehat{\Psi}\right|_{\Gamma_{10}},\left.\frac{\partial \widehat{\Psi}}{\partial n}\right|_{\Gamma_{8}}=\left.\frac{\partial \widehat{\Psi}}{\partial n}\right|_{\Gamma_{10}}$ at $\Gamma_{8}$ and $\Gamma_{10}$.

We consider the shape parameter is $2.4 \times 10^{-3}$. The boundary and inner point numbers are 360 and 243, respectively as depicted in Fig. 10 (b). Fig. 11 demonstrates the profile of the computed pressure head in a three-layered unsaturated soil. In this example, infiltration starts in the high permeable soil layer (layer C). It is found that infiltration reaches the top side of the less permeable soil layer (layer B), the pressure head at the interface increases rapidly so that infiltration can flow through the less permeable soil layer (layer B). However, 


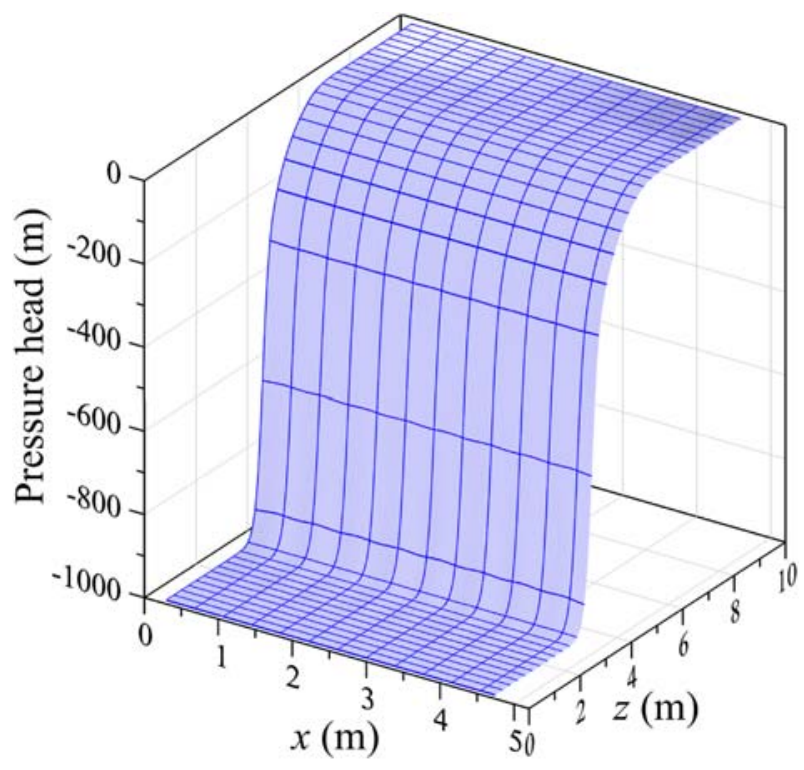

Fig. 11. The computed results for the two-dimensional infiltration problem.

when the amount of unsaturated flows reach the interface with the high permeable soil (layer A), the amount of unsaturated flows to layer A may be dissipated easily due to the high conductivity of the layer A, as depicted in Fig. 12.

\subsection{Two-dimensional infiltration process in an unsaturated multi-layered soil}

The last application example under investigation is a two-dimensional infiltration problem in multi-layered soil, as depicted in Fig. 13 (a). The length and the height of the soil are considered to be 20 and $90 \mathrm{~m}$. In the case of multi-layered soil, we consider the thickness of layer A, layer B, layer C, layer $D$, layer $E$, layer $F$ and layer $G$ to be $8,6,14,9$, 10, 10, and $33 \mathrm{~m}$, as shown in Fig. 13 (a).

In this example, the soil type of layer A and layer $\mathrm{D}$ are considered to be coarse sand, the soil type of layer $B$ and layer $G$ are considered to be fine sand, the soil type of layer $C$ and layer $E$ are considered to be clean gravel, and the soil type of layer $\mathrm{F}$ are considered to be clay. We consider the saturated permeability of clean gravel, coarse sand, fine sand, and clay to be $10^{-2}, 10^{-4}, 10^{-5}$ and $10^{-9} \mathrm{~m} / \mathrm{s}$, respectively. The parameters for saturated

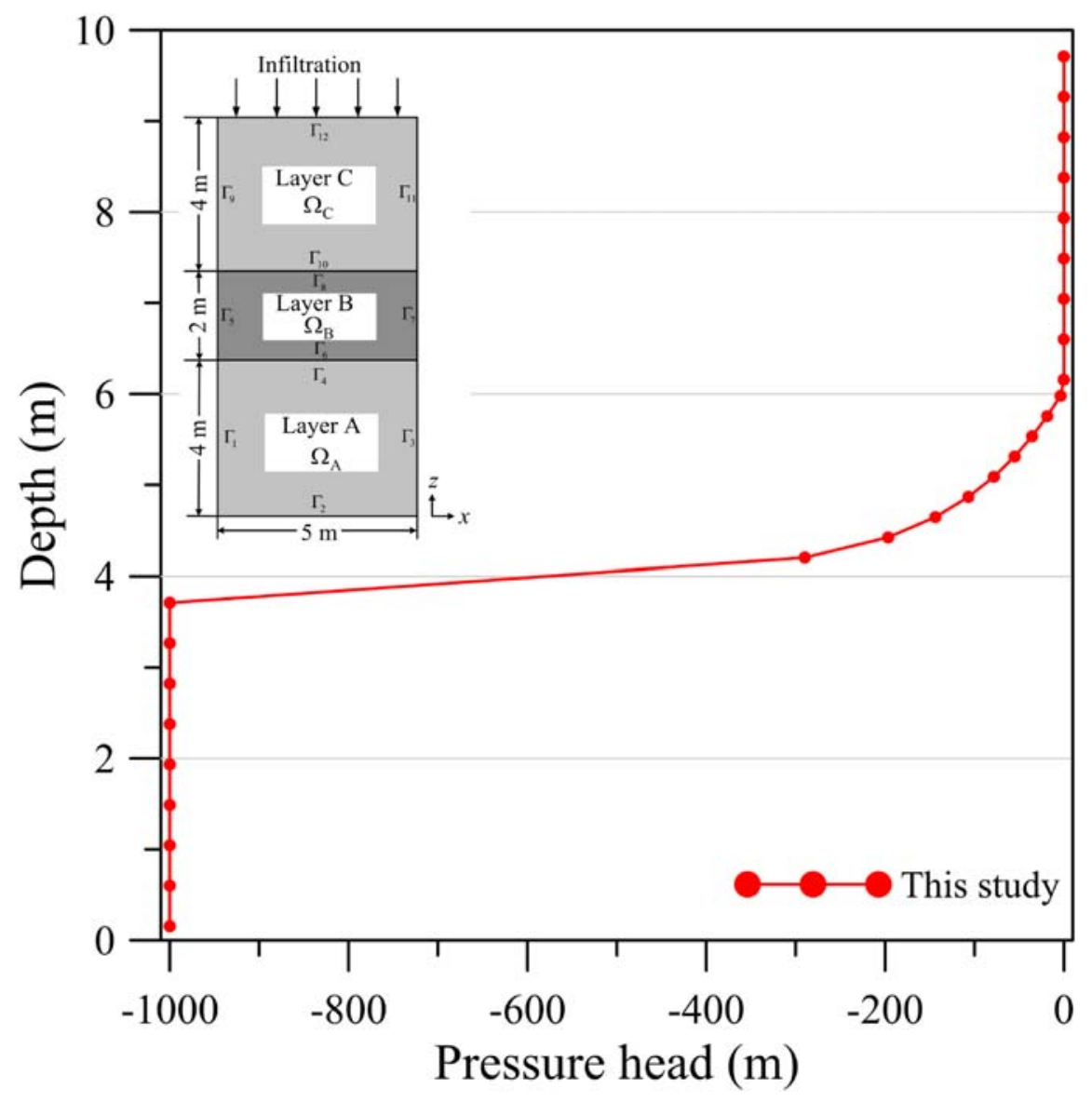

Fig. 12. The pressure head profiles for the two-dimensional infiltration problem. 


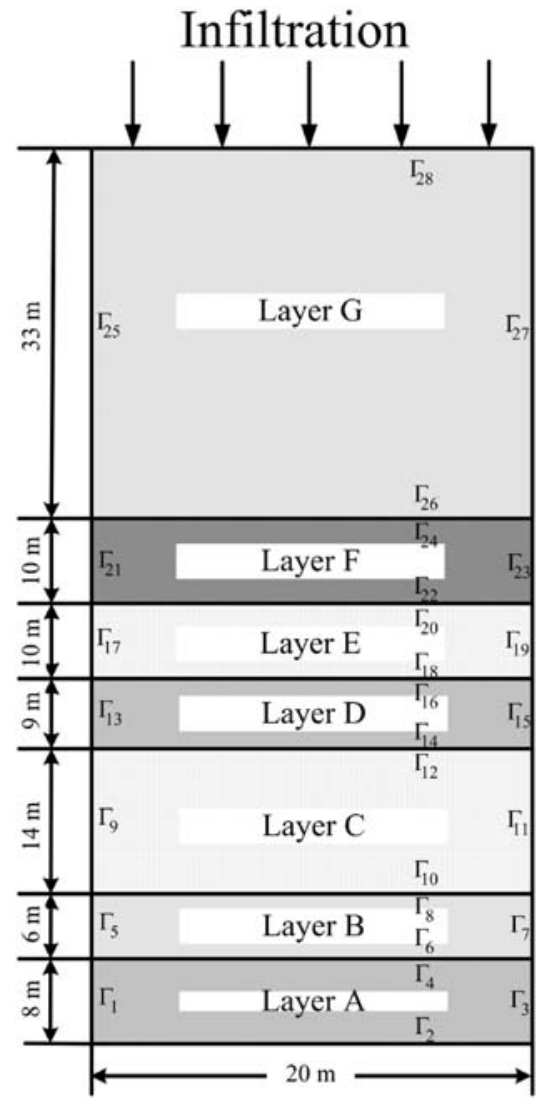

(a) Illustration of the infiltration problem in an unsaturated multi-layered soil.
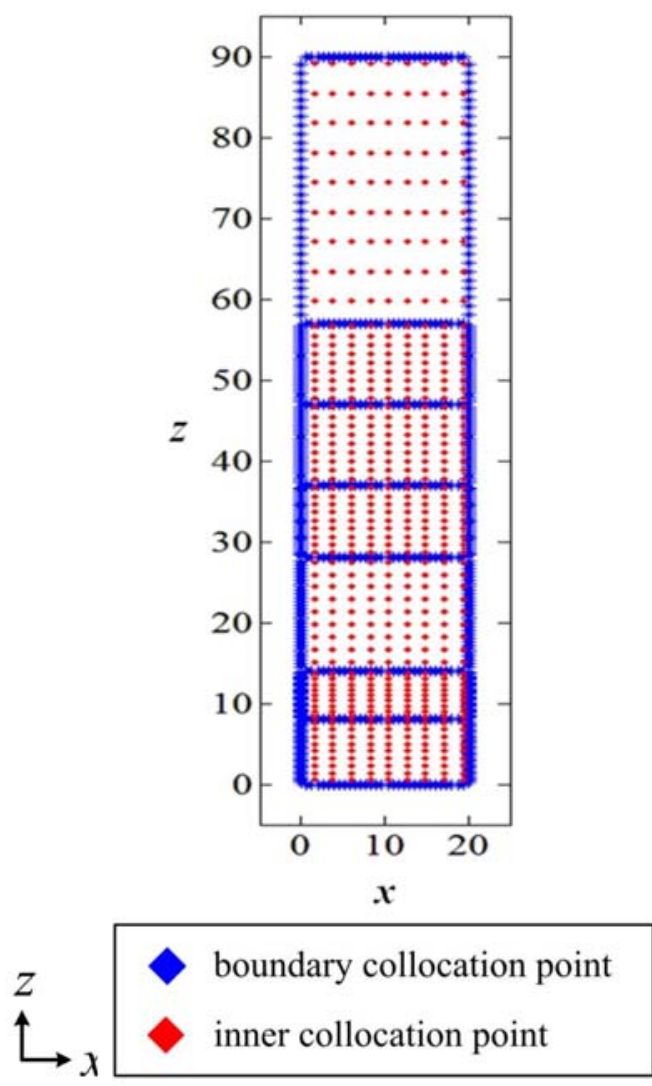

(b) Points collocated on the boundary and within the domain.

Fig. 13. Illustration of example 4.3.

permeability of soils are listed in Table 3. The pore size distribution parameter, the saturated and residual moisture contents of each layer are set to be $4 \times 10^{-3}, 0.46$, and 0.14 .

The boundary conditions including $\Gamma_{1}, \Gamma_{2}, \ldots, \Gamma_{28}$ are shown in Fig. 13 (a). The top and bottom boundaries are Dirichlet boundary conditions and the lateral boundary condition to be no-flow imperious boundary condition, which can be written as following equations.

Table 3. Unsaturated soil parameters.

\begin{tabular}{lll}
\hline layer & soil type & permeability $(\mathrm{m} / \mathrm{s})$ \\
\hline layer A & coarse sand & $10^{-4}$ \\
layer B & fine sand & $10^{-5}$ \\
layer C & clean gravel & $10^{-2}$ \\
layer D & coarse sand & $10^{-4}$ \\
layer E & clean gravel & $10^{-2}$ \\
layer F & clay & $10^{-9}$ \\
layer G & fine sand & $10^{-5}$ \\
\hline
\end{tabular}

$\widehat{\Psi}(x, z=L)=\left(1-e^{\alpha \Psi_{d}}\right)$ on $\Gamma_{28}$,

$\widehat{\Psi}(x, z=0)=0$ on $\Gamma_{2}$,

$\frac{\partial \widehat{\Psi}(x=0, z)}{\partial z}=0$ on $\Gamma_{1}, \Gamma_{5}, \Gamma_{9}, \Gamma_{13}, \Gamma_{17}, \Gamma_{21}$ and $\Gamma_{25}$,

$\frac{\partial \widehat{\Psi}(x=a, z)}{\partial z}=0$ on $\Gamma_{3}, \Gamma_{7}, \Gamma_{11}, \Gamma_{15}, \Gamma_{19}, \Gamma_{23}$ and $\Gamma_{27}$

At interfaces, the boundary conditions must satisfy the following equations.

$\left.\widehat{\Psi}\right|_{\Gamma_{4}}=\left.\widehat{\Psi}\right|_{\Gamma_{6}},\left.\frac{\partial \widehat{\Psi}}{\partial n}\right|_{\Gamma_{4}}=\left.\frac{\partial \widehat{\Psi}}{\partial n}\right|_{\Gamma_{6}}$ at $\Gamma_{4}$ and $\Gamma_{6}$,

$\left.\widehat{\Psi}\right|_{\Gamma_{8}}=\left.\widehat{\Psi}\right|_{\Gamma_{10}},\left.\frac{\partial \widehat{\Psi}}{\partial n}\right|_{\Gamma_{8}}=\left.\frac{\partial \widehat{\Psi}}{\partial n}\right|_{\Gamma_{10}}$ at $\Gamma_{8}$ and $\Gamma_{10}$, 


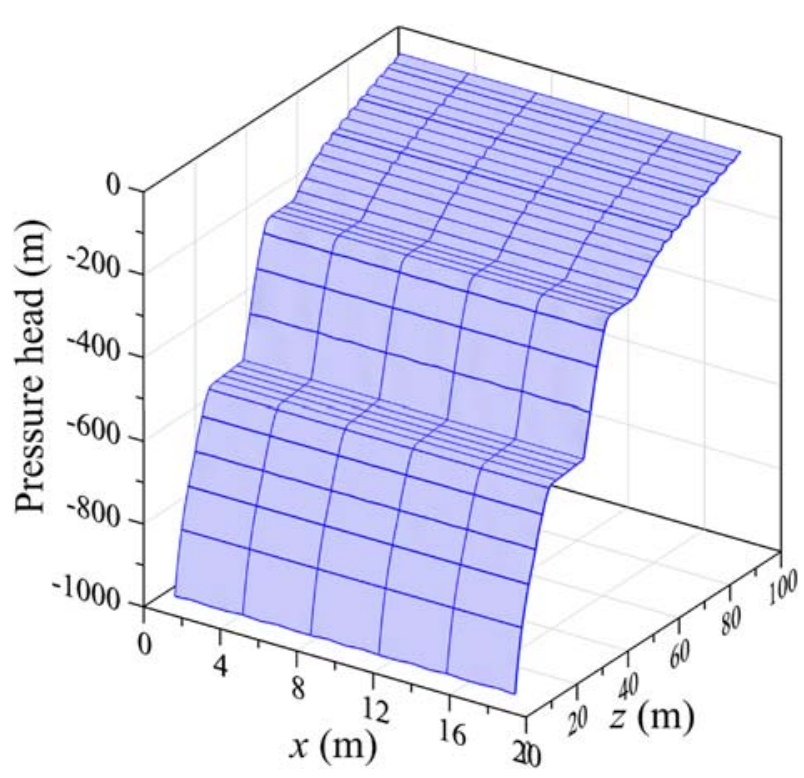

Fig. 14. The computed pressure head distribution for the two-dimensional infiltration problem.
$\left.\widehat{\Psi}\right|_{\Gamma_{12}}=\left.\widehat{\Psi}\right|_{\Gamma_{14}},\left.\frac{\partial \widehat{\Psi}}{\partial n}\right|_{\Gamma_{12}}=\left.\frac{\partial \widehat{\Psi}}{\partial n}\right|_{\Gamma_{14}}$ at $\Gamma_{12}$ and $\Gamma_{14}$

$\left.\widehat{\Psi}\right|_{\Gamma_{16}}=\left.\widehat{\Psi}\right|_{\Gamma_{18}},\left.\frac{\partial \widehat{\Psi}}{\partial n}\right|_{\Gamma_{16}}=\left.\frac{\partial \widehat{\Psi}}{\partial n}\right|_{\Gamma_{18}}$ at $\Gamma_{16}$ and $\Gamma_{18}$

$\left.\widehat{\Psi}\right|_{\Gamma_{20}}=\left.\widehat{\Psi}\right|_{\Gamma_{22}},\left.\frac{\partial \widehat{\Psi}}{\partial n}\right|_{\Gamma_{20}}=\left.\frac{\partial \widehat{\Psi}}{\partial n}\right|_{\Gamma_{22}}$, at $\Gamma_{20}$ and $\Gamma_{22}$,

$\left.\widehat{\Psi}\right|_{\Gamma_{24}}=\left.\widehat{\Psi}\right|_{\Gamma_{26}},\left.\frac{\partial \widehat{\Psi}}{\partial n}\right|_{\Gamma_{24}}=\left.\frac{\partial \widehat{\Psi}}{\partial n}\right|_{\Gamma_{26}}$, at $\Gamma_{24}$ and $\Gamma_{26}$.

In this example, the shape parameter is $2.4 \times$ $10^{-3}$. The boundary and inner point numbers are 840 and 567, respectively as depicted in Fig. 13 (b). Fig. 14 depicts the profile of the pressure head in a multi-layered unsaturated soil computed by the proposed method.

It is found that our proposed method can still achieve reasonable results even if the infiltration problem in the multi-layered soil with different

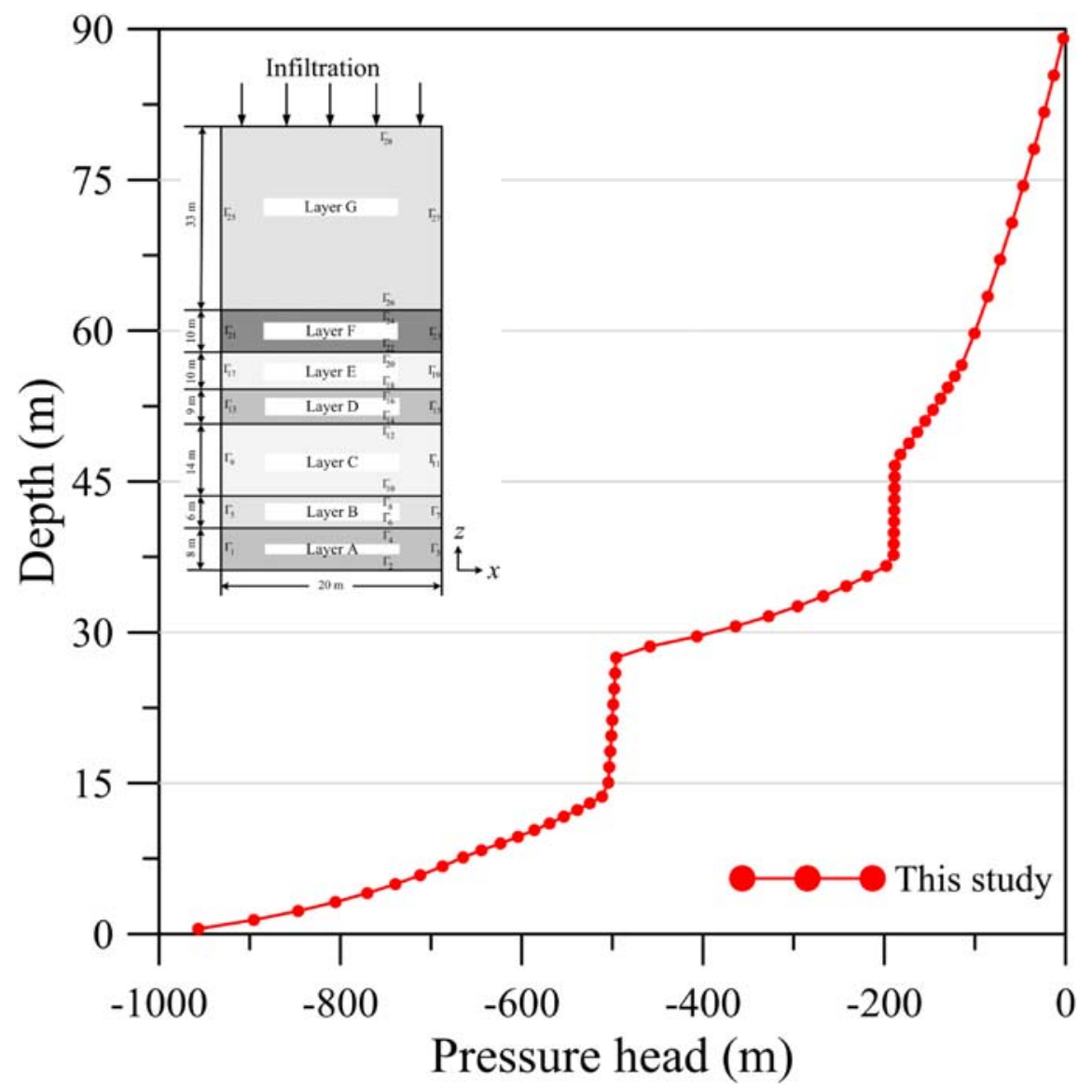

Fig. 15. The pressure head profiles for the two-dimensional infiltration problem. 
physical property contrasts is considered, as depicted in Fig. 15. The results further demonstrate that the method could be implemented to simulate two-dimensional infiltration process in the unsaturated multi-layered soil with large physical contrasts in permeability.

\section{Conclusions}

In this study, we propose a meshless method with the RBF for solving unsaturated layered heterogeneous flow in two dimensions. An exponential model is introduced in the Richards equation such that we obtain the linearized Richards equation for the unsaturated soil. Numerical implementations are established to validate the proposed method. Several application examples are carried out for modeling the two-dimensional hydrological process in the multi-layered unsaturated soil. Findings from this article are summarized as follows.

1. The meshless method with the RBF for solving flow movement in unsaturated layered heterogeneous soils using the Richards equation with the exponential model is developed. Our method may provide an alternative from other mesh-based methods for modeling the appearance of unsaturated layered heterogeneous soils.

2. The validity of the proposed method is achieved for several numerical implementations including one- and two-dimensional unsaturated flow problems. Our proposed method can simulate the hydrological process in unsaturated layered soils with high accuracy. Furthermore, numerical results indicate that the method can easily implement unsaturated multi-layered problems with large contrasts in which the problems are usually ill-posed and difficult to be solved in the past.

3. Results obtained demonstrate that the proposed approach utilizing the Richards equation with the Gardner exponential model is highly accurate to deal with unsaturated flow in heterogeneous porous media in two dimensions. It is expected that the findings obtained in this study may be applicable for solving the three-dimensional unsaturated flow problems in heterogeneous porous media.

\section{Acknowledgments}

This study is partially supported by the Ministry of Science and Technology, Taiwan, the Republic of
China (MOST 108-2621-M-019-008). The authors thank the Ministry of Science and Technology for the generous financial support. The first author is also grateful to his former student, Pei-Rong Li, for her assistance of this study.

\section{References}

[1] Chan HF, Fan CM. The local radial basis function collocation method for solving two-dimensional inverse Cauchy problems. Numer Heat Tran, Part B: Fundamentals 2013;63: 284-303.

[2] Chen S, Liu F, Burrage K. Numerical simulation of a new two-dimensional variable-order fractional percolation equation in non-homogeneous porous media. Comput Math Appl 2014;68:2133-41.

[3] Chen X, Dai Y. An approximate analytical solution of Richards' equation. Int J Nonlinear Sci Numer Stimul 2015; 16.

[4] Chen X, Dai Y. Wetting front analysis of the Richards equation with impervious boundary. Int J Nonlinear Sci Numer Stimul 2018;19:595-604.

[5] Dehghan M, Mohammadi V. The numerical solution of Fokker-Planck equation with radial basis functions (RBFs) based on the meshless technique of Kansa's approach and Galerkin method. Eng Anal Bound Elem 2014;47:38-63.

[6] Di Prima S, Castellini M, Pirastru M, Keesstra S. Soil water conservation: dynamics and impact. Water 2018;10:952.

[7] Driscoll TA, Heryudono ARH. Adaptive residual subsampling methods for radial basis function interpolation and collocation problems. Comput Math Appl 2007;53: 927-39.

[8] Farcas A, Elliott L, Ingham DB, Lesnic D. An inverse dual reciprocity method for hydraulic conductivity identification in steady groundwater flow. Adv Water Resour 2004;27: 223-35.

[9] Farthing MW, Ogden FL. Numerical solution of Richards' equation: a review of advances and challenges. Soil Sci Soc Am J 2017;81:1257-69.

[10] Gardner WR. Some steady-state solutions of the unsaturated moisture flow equation with application to evaporation from a water table. Soil Sci 1958;85:228-32.

[11] Hanoglu U, Sarler B. Simulation of hot shape rolling of steel in continuous rolling mill by local radial basis function collocation method. Comp Model Eng Sci 2015;109-110: 447-79.

[12] Jankowska MA, Karageorghis A, Chen CS. Improved Kansa RBF method for the solution of nonlinear boundary value problems. Eng Anal Bound Elem 2018;87:173-83.

[13] Johansson BT, Lesnic D. A method of fundamental solutions for transient heat conduction. Eng Anal Bound Elem 2008;32: 697-703.

[14] Kansa E. Multiquadrics-A scattered data approximation scheme with applications to computational fluid dynamics-I Surface approximations and partial derivative estimates. Comput Math Appl 1990;19:127-45.

[15] Ku CY, Liu CY, Su Y, Xiao JE, Yeih W. Transient modeling of flow in unsaturated soils using a novel collocation meshless method. Water 2017;9:954.

[16] Li M, Jiang T, Hon YC. A meshless method based on RBFs method for nonhomogeneous backward heat conduction problem. Eng Anal Bound Elem 2010;34:785-92.

[17] Lin DG, Hung SH, Liu WT, Chou JC. Evaluation on the efficiency of stability remediation for t16-tower pier slope (t16slope) of taipei mao-kong tramway. J Mar Sci Technol 2018; 26:352-73.

[18] Liu CS, Chang CW. An energy regularization of the MQ-RBF method for solving the Cauchy problems of diffusion-convection-reaction equations. Commun Nonlinear Sci Numer Simulat 2019;67:375-90. 
[19] Liu CS, Liu D. Optimal shape parameter in the MQ-RBF by minimizing an energy gap functional. Appl Math Lett 2018; 86:157-65.

[20] Liu CY, Ku CY, Huang CC, Lin DG, Yeih W. Numerical solutions of groundwater flow in unsaturated layered soil with extreme physical property contrasts. Int J Nonlinear Sci Numer Stimul 2015;16:325-36.

[21] Liu CY, Ku CY, Xiao JE, Huang CC, Hsu SM. Numerical modeling of unsaturated layered soil for rainfall-induced shallow landslides. J Environ Eng Landsc Manag 2017;25: $329-41$.

[22] Liu CY, Ku CY, Xiao JE, Yeih W. A novel spacetime collocation meshless method for solving two-dimensional backward heat conduction problems. Comput Model Eng Sci 2019;118:229-52

[23] Mavrič B, Sarler B. Application of the RBF collocation method to transient coupled thermoelasticity. Int J Numer Methods Heat Fluid Flow 2017;27:1064-77.

[24] Mohamed S, Ben-Ahmed EH, Wakrim M. RBFPUM with QR factorization for solving water flow problem in multilayered soil. Int J Nonlinear Sci Numer Stimul 2018;19:397-408.

[25] Motaman F, Rakhshandehroo GR, Hashemi MR, Niazkar M. Application of RBF-DQ method to time-dependent analysis of unsaturated seepage. Transport Porous Media 2018;125: $543-64$.

[26] Rafiezadeh K, Ataie-Ashtiani B. Transient free-surface seepage in three-dimensional general anisotropic media by BEM. Eng Anal Bound Elem 2014;46:51-66.

[27] Sarra SA. Adaptive radial basis function methods for time dependent partial differential equations. Appl Numer Math 2005;54:79-94.

[28] Srivastava R, Yeh TCJ. Analytical solutions for one-dimensional, transient infiltration toward the water table in homogeneous and layered soils. Water Resour Res 1991;27: 753-62.

[29] Stevens D, Power H. A scalable and implicit meshless RBF method for the 3D unsteady nonlinear Richards equation with single and multi-zone domains. Int J Numer Methods Eng 2011;85:135-63.

[30] Stevens D, Power H, Lees M, Morvan H. A meshless solution technique for the solution of $3 \mathrm{D}$ unsaturated zone problems, based on local Hermitian interpolation with radial basis functions. Transport Porous Media 2009;79: 149-69.

[31] Toufigh V. Constrained optimization based F.E. mesh deforming algorithm for unconfined seepage problems. Appl Math Model 2016;40:6754-65.

[32] Tracy FT. Clean two- and three-dimensional analytical solutions of Richards' equation for testing numerical solvers. Water Resour Res 2006;42:W08503.

[33] Wang BL, Mai YW. Transient one-dimensional heat conduction problems solved by finite element. Int J Mech Sci 2005;47:303-17.

[34] Wang H, Li J. Analytical solutions to the one-dimensional coupled seepage and deformation of unsaturated soils with arbitrary nonhomogeneous boundary conditions. Transport Porous Media 2015;108:481-96.

[35] Wang J, Su L, Qin X. Hermite radial basis collocation method for unsaturated soil water movement equation. Math Probl Eng 2018;2018:8298915.

[36] Xiao JE, Ku CY, Liu CY, Yeih WC. A novel boundary-type meshless method for modeling geofluid flow in heterogeneous geological media. Geofluids 2018;2018:9804291.

[37] Yang JP, Su WT. Investigation of radial basis collocation method for incremental-iterative analysis. Int J Appl Mech 2016;8:1650007. 